\title{
ON THE DIMENSION OF THE HILBERT SCHEME OF CURVES
}

\author{
DAWEI Chen
}

\begin{abstract}
Consider an irreducible component of the Hilbert scheme whose general points parameterize degree $d$ genus $g$ smooth irreducible and non-degenerate curves in a projective variety $X$. We give lower bounds for the dimension of such components when $X$ is $\mathbb{P}^{3}, \mathbb{P}^{4}$ or a smooth quadric threefold in $\mathbb{P}^{4}$, respectively. Those bounds make sense from the asymptotic viewpoint if we fix $d$ and let $g$ vary. Some examples are constructed using determinantal varieties to show the sharpness of the bounds for $d$ and $g$ in a certain range. The results can be applied to study rigid curves.
\end{abstract}

\section{Introduction}

In this section, we briefly recall some facts about Hilbert schemes, and state the main results of this paper. We always work over an algebraically closed field of characteristic zero.

Let $P$ be the Hilbert polynomial of a subscheme in $\mathbb{P}^{r}$. We can ask if there exists a moduli space parameterizing all subschemes of $\mathbb{P}^{r}$ that have $P$ as their Hilbert polynomial. Grothendieck [7] proved there exists a fine moduli space $\operatorname{Hilb}^{P}\left(\mathbb{P}^{r}\right)$. Moreover, it is a projective scheme. Few facts about the global properties of the Hilbert scheme $\operatorname{Hilb}^{P}\left(\mathbb{P}^{r}\right)$ have been obtained. Nevertheless, Hartshorne [11] proved that $\operatorname{Hilb}^{P}\left(\mathbb{P}^{r}\right)$ is connected if not empty.

Here curves are our main interests. By a curve, we mean a purely 1-dimensional closed subscheme in $\mathbb{P}^{r}$. The Hilbert polynomial $P$ of a curve is a linear function with leading coefficient $d$ and constant term $1-g$, i.e. $P(m)=d m+1-g$, where $d$ and $g$ are the degree and arithmetic genus of the curve. Hilb ${ }^{d m+1-g}\left(\mathbb{P}^{r}\right)$ may have many irreducible components even for small $r$. For instance, it was shown [4] that the number of irreducible components of Hilb ${ }^{d m+1-g}\left(\mathbb{P}^{3}\right)$ cannot be bounded by a polynomial of $d$ and $g$.

In this paper we study the dimension of $\mathrm{Hilb}^{d m+1-g}\left(\mathbb{P}^{r}\right)$. A well-known result is the following, cf. e.g. [10, 1.E].

Theorem 1.1. Let $C$ be a curve in $\mathbb{P}^{r}$ such that $[C] \in H i l b^{d m+1-g}\left(\mathbb{P}^{r}\right)$. The tangent space of Hilb ${ }^{d m+1-g}\left(\mathbb{P}^{r}\right)$ at $[C]$ can be identified as

$$
T_{[C]}=H^{0}\left(C, N_{C / \mathbb{P}^{r}}\right),
$$

where $N_{C / \mathbb{P}^{r}}$ is the normal sheaf of $C$ in $\mathbb{P}^{r}$. Moreover, if $C$ is a locally complete intersection, then

$$
\chi\left(N_{C / \mathbb{P}^{r}}\right) \leq \operatorname{dim}_{[C]} H_{i l b}{ }^{d m+1-g}\left(\mathbb{P}^{r}\right) \leq h^{0}\left(C, N_{C / \mathbb{P}^{r}}\right),
$$

Received by the editors August 28, 2008.

2000 Mathematics Subject Classification. Primary: 14C05, 14H10, 14H50.

Part of the work was done when the author was supported by the 2008 Clay Liftoff Fellowship. 
where $\chi\left(N_{C / \mathbb{P}^{r}}\right)=h^{0}\left(C, N_{C / \mathbb{P}^{r}}\right)-h^{1}\left(C, N_{C / \mathbb{P}^{r}}\right)$.

Definition 1.2. Let $U$ be an irreducible component of Hilb ${ }^{d m+1-g}\left(\mathbb{P}^{r}\right)$ whose general points parameterize smooth irreducible and non-degenerate curves in $\mathbb{P}^{r}$. Define $l_{d, g, r}$ as the lowest dimension of all such components $U$.

The goal of this paper is to bound $l_{d, g, r}$ from below. For a locally complete intersection $[C] \in \operatorname{Hilb}^{d m+1-g}\left(\mathbb{P}^{r}\right)$, we have $\chi\left(N_{C / \mathbb{P}^{r}}\right)=(r+1) d-(r-3)(g-1)$, cf. [10, 1.44]. Based on Theorem 1.1, we know that $l_{d, g, r} \geq \chi\left(N_{C / \mathbb{P}^{r}}\right)$. However, this lower bound $\chi\left(N_{C / \mathbb{P}^{r}}\right)$ is not sharp in a number of cases.

For the beginning case $r=3, \chi\left(N_{C / \mathbb{P}^{r}}\right)=4 d$ is independent of $g$. If we fix $d$ and let $g$ vary, the genus of a degree $d$ reduced irreducible and non-degenerate curve in $\mathbb{P}^{3}$ can be as large as the Castelnuovo bound $\pi(d, 3)=\frac{d^{2}}{4}+O(d)$. Fix $d$ and let $g$ vary. When $g$ approaches its maximum $\pi(d, 3)$, we have $l_{d, g, 3}=g+O(d)$, which can be much larger than $4 d$. One can refer to $[9$, Ch. 3] for these results on the Castelnuovo theory. Our first result is the following improved lower bound for $l_{d, g, 3}$.

Theorem 1.3. When $g^{2} \geq d^{3}$, define $\mu(d, g)$ as the smallest integer that is greater than

$$
1+\frac{d^{2}-4 d-4 g}{g+\sqrt{g^{2}-d^{3}+4 d g+4 d^{2}}} .
$$

Then for any $d \geq 3$ and $d \sqrt{d} \leq g \leq \pi(d, 3)$, we have

$$
l_{d, g, 3} \geq 4 d+g-1-\mu(d, g) d .
$$

For fixed $d$, the bound $4 d+g-1-\mu(d, g) d$ is increasing with $g$. In particular, it goes to $g+O(d)$ when $g$ approaches the Castelnuovo bound $\pi(d, 3)$.

The lower bound involved in Theorem 1.3 may look confusing. Nevertheless, let us consider an example to show the power of this bound. Suppose $d=100$ and $g$ varies from 0 to the Castelnuovo bound $\pi(100,3)=2401$. Pick $g=1100$, which is large but not close to the Castelnuovo bound. The bound $4 d$ only says that $l_{100,1100,3} \geq 400$. However, by Theorem 1.3 , we get $l_{100,1100,3} \geq 1099$, which is much better.

Now consider the case $r \geq 4$. The number $\chi\left(N_{C / \mathbb{P}^{r}}\right)=(r+1) d-(r-3)(g-1)$ could be negative if $g$ is larger than $d$. So it makes sense to find at least a positive lower bound for $l_{d, g, r}$. Furthermore, such a lower bound may also help answer a question regarding rigid curves.

Definition 1.4. A rigid curve in $\mathbb{P}^{r}$ is a smooth irreducible and non-degenerate curve that does not have deformations except those induced from the automorphisms of $\mathbb{P}^{r}$.

For instance, a rational normal curve is rigid. To the author's best knowledge, people have not found any other rigid curves. Harris and Morrison [10, 1.47] conjectured that there does not exist a rigid curve except rational normal curves. One way to attack this conjecture is to bound $l_{d, g, r}$ from below. If the inequality $l_{d, g, r}>\operatorname{dim}$ $\operatorname{PGL}(r)=r^{2}+2 r$ holds, then there cannot exist a degree $d$ genus $g$ rigid curve in $\mathbb{P}^{r}$. In fact, this is one of our motivations to study $l_{d, g, r}$.

For the case $r=4$, we have the following result.

Theorem 1.5. Let $C$ be a degree $d$ genus $g$ smooth irreducible and non-degenerate curve in $\mathbb{P}^{4}$. Fix $d \geq 5$ and let $g$ vary. If $g>3 d \sqrt{d}+O(d)$, then $C$ is not rigid. 
Here we could be more precise on the range of $d$ and $g$ as we have done in Theorem 1.3. However, we choose to only focus on the asymptotic behavior, since the order $d \sqrt{d}$ seems to be important. Currently we have not been able to extend the result to $r \geq 5$. But combining the results in [2], we expect the following conjecture to hold in general.

Conjecture 1.6. For $r \geq 5$, there exists a constant $\lambda_{r}$ such that if $g \geq \lambda_{r} d \sqrt{d}+O(d)$, a degree $d$ genus $g$ smooth irreducible and non-degenerate curve in $\mathbb{P}^{r}$ is not rigid.

In addition to projective spaces, we can also study the deformation of curves on a hypersurface. The beginning case would be a smooth quadric threefold in $\mathbb{P}^{4}$. Since all the smooth quadrics in $\mathbb{P}^{4}$ are isomorphic, we fix one and denote it by $Q$.

Definition 1.7. Let $U$ be an irreducible component of $\operatorname{Hilb}^{d m+1-g}(Q)$ whose general points parameterize smooth irreducible and non-degenerate curves on $Q$. Define the number $q_{d, g}$ as the smallest dimension of all such components $U$.

By the short exact sequence

$$
\left.0 \rightarrow N_{C / Q} \rightarrow N_{C / \mathbb{P}^{4}} \rightarrow N_{Q / \mathbb{P}^{4}}\right|_{C} \rightarrow 0,
$$

it follows that $\chi\left(N_{C / Q}\right)=3 d$. So $3 d$ provides a lower bound for $q_{d, g}$. We can still ask how good this lower bound could be. A similar result as Theorem 1.5 can be established as follows.

Theorem 1.8. Fix $d$ and let $g$ vary.

(1) If $g>\frac{1}{\sqrt{2}} d \sqrt{d}+O(d)$, then $q_{d, g}$ is strictly greater than $3 d$.

(2) If $g<\frac{2}{15 \sqrt{5}} d \sqrt{d}+O(d)$, the equality $q_{d, g}=3 d$ holds.

Again, we only focus on the asymptotic behavior. The coefficients of $d \sqrt{d}$ might be improved by refining our techniques, but the order $d \sqrt{d}$ seems to be correct.

\section{The Hilbert scheme of curves in $\mathbb{P}^{3}$}

In this section, we will firstly prove Theorem 1.3. Fix $d$ and let $g$ vary in the range $g^{2} \geq d^{3}$. The upshot is that for large $g$, a curve must be contained in a low degree surface, cf. Theorem 2.1. We can estimate the dimension of the deformation of the curve on that surface, which provides a better bound than $4 d$. Then we will discuss whether the expected dimension $4 d$ is sharp when $g^{2}<d^{3}$.

Let us start from a result originally mentioned by Halphen and proved later by Gruson and Peskine [8].

Theorem 2.1. Let $C$ be a connected smooth curve of degree $d$ and genus $g$ in $\mathbb{P}^{3}$. Let $t$ denote a positive integer such that $t(t-1)<d$. If $g$ satisfies

$$
g>\frac{d}{2}\left(t+\frac{d}{t}-4\right)-\frac{r(t-r)(t-1)}{2 t},
$$

where $0 \leq r<t, d+r \equiv 0(\bmod t)$, then $C$ must lie on a surface of degree $\leq t$.

Note that if $t \sim \sqrt{d}$, then the right hand side of $(1) \sim d \sqrt{d}$. Hence, Theorem 2.1 can help us deal with the case $g^{2}>d^{3}$. For computational convenience, Theorem 2.1 can be slightly modified as follows. 
Proposition 2.2. Let $C$ be a connected smooth curve of degree $d$ and genus $g$ in $\mathbb{P}^{3}$. Let $s$ denote a positive integer such that $s(s+1)<d$. If $g$ satisfies

$$
g>\frac{d}{2}\left(s+\frac{d}{s+1}-3\right),
$$

then $C$ must lie on a surface of degree $k \leq s$.

Proof. In Theorem 2.1, let $t=s+1$.

For fixed $d$ and $g$ in the range $g^{2} \geq d^{3}$, consider the smallest positive integer $s$ satisfying $s(s+1)<d$ and the inequality (2). By Proposition 2.2, there exists a surface $S$ of degree $k \leq s$ such that $S$ contains $C$. Let Hilb ${ }^{d m+1-g}(S)$ be the Hilbert scheme parameterizing degree $d$ and arithmetic genus $g$ curves on $S$. Hilb $^{d m+1-g}(S)$ can be viewed as a subscheme of Hilb ${ }^{d m+1-g}\left(\mathbb{P}^{3}\right)$. We want to estimate $\operatorname{dim}_{[C]} \operatorname{Hilb}^{d m+1-g}(S)$.

If $S$ is smooth, then $\chi\left(N_{C / S}\right)$ provides a lower bound for $\operatorname{dim}_{[C]} \operatorname{Hilb}^{d m+1-g}(S)$. We have the short exact sequence

$$
0 \rightarrow N_{C / S} \rightarrow N_{C / \mathbb{P}^{3}} \rightarrow N_{S / \mathbb{P}^{3}} \otimes \mathcal{O}_{C} \rightarrow 0 .
$$

By the adjunction formula, $N_{S / \mathbb{P} 3} \otimes \mathcal{O}_{C}=\mathcal{O}_{C}(k)$. Then we can compute $\chi\left(N_{C / S}\right)$ by the exact sequence (3) and Riemann-Roch,

$$
\begin{aligned}
\chi\left(N_{C / S}\right) & =\chi\left(N_{C / \mathbb{P}^{3}}\right)-\chi\left(N_{S / \mathbb{P}^{3}} \otimes \mathcal{O}_{C}\right) \\
& =4 d-\chi\left(\mathcal{O}_{C}(k)\right) \\
& =4 d+g-1-k d \\
& \geq 4 d+g-1-s d .
\end{aligned}
$$

It follows that

$$
\operatorname{dim}_{[C]} \operatorname{Hilb}^{d m+1-g}\left(\mathbb{P}^{3}\right) \geq \operatorname{dim}_{[C]} \operatorname{Hilb}^{d m+1-g}(S) \geq 4 d+g-1-s d .
$$

Therefore, we get a lower bound for the dimension of Hilb ${ }^{d m+1-g}\left(\mathbb{P}^{3}\right)$,

$$
l_{d, g, 3} \geq 4 d+g-1-s d .
$$

The advantage of (4) is because in the range $g^{2} \geq d^{3}$, as $g$ increases, $s$ decreases, and $4 d+g-1-s d$ is more dominated by $g$. For instance, if we fix $d$ and let $g$ approach the Castelnuovo bound $\pi(d, 3)$, then $l_{d, g, 3}$ tends to $g$. But at this moment $s$ is very small. Therefore, the estimate (4) does not lose much information from the asymptotic viewpoint.

In order to establish (4) when the surface $S$ is singular and $C$ passes through singular points of $S$, we have to use Ext groups instead of cohomology. Before doing that, let us prove a simple result, which shows that the situation is not very bad even if $S$ is singular.

Lemma 2.3. Let $S_{\text {sing }}$ denote the singular locus of the surface $S$. Under the assumption of Proposition 2.2, if $C \cap S_{\text {sing }}$ is not empty, then it is 0-dimensional.

Proof. If the dimension of $S_{\text {sing }}$ is 0 , then the statement is trivial. Otherwise the dimension of $S_{\text {sing }}$ is 1 . By Bézout, the degree of $S_{\text {sing }}$ is at most $k(k-1) \leq s(s-1)<$ $d$, where $k$ is the degree of $S$. Hence, $C$ cannot be contained in $S_{\text {sing }}$.

By Lemma 2.3, we can apply the following result from [14, Ch. 1, 2.13, 2.15]. 
Proposition 2.4. Keep the above notation. If $C \cap S_{\text {sing }}$ is 0-dimensional, then $C \subset S$ is generically unobstructed and the dimension of every irreducible component of $\mathrm{Hilb}^{\mathrm{dm+1-g}}(\mathrm{S})$ at $[\mathrm{C}]$ is at least

$$
\operatorname{dim} \operatorname{Hom}_{C}\left(I_{C / S} / I_{C / S}^{2}, \mathcal{O}_{C}\right)-\operatorname{dim} \operatorname{Ext}_{C}^{1}\left(I_{C / S} / I_{C / S}^{2}, \mathcal{O}_{C}\right) .
$$

Note that for smooth surface $S$, the value of (5) is just $\chi\left(N_{C / S}\right)$. When $S$ is singular, we need to verify some exact sequences of Kähler differentials. We will do it in a more general setting since the results can be applied to many other cases.

Proposition 2.5. Let $C$ be a smooth connected curve and $X$ be an $(n-k)$-dimensional locally complete intersection such that $C \subset X \subset \mathbb{P}^{n}, n \geq 3,1 \leq k \leq n-2$. If $C \cap X_{\text {sing }}$ is 0-dimensional, we have the following exact sequences

$$
\begin{array}{r}
0 \rightarrow I_{C / X} / I_{C / X}^{2} \stackrel{d}{\rightarrow} \Omega_{X} \otimes \mathcal{O}_{C} \rightarrow \Omega_{C} \rightarrow 0, \\
0 \rightarrow\left(I_{X} / I_{X}^{2}\right) \otimes \mathcal{O}_{C} \stackrel{d}{\rightarrow} \Omega_{\mathbb{P}^{n}} \otimes \mathcal{O}_{C} \rightarrow \Omega_{X} \otimes \mathcal{O}_{C} \rightarrow 0 .
\end{array}
$$

Note that if $X$ is smooth, these results are well-known. When $X$ is singular, the above sequences are still exact except the left hand sides may not be injective, cf. [12, Ch. II 8].

Proof. It suffices to verify that the map to the middle term is injective for each sequence. Since the question is local, we only need to work on a local affine chart $U$. Suppose $x_{1}, \ldots, x_{n}$ are the local coordinates, and $f_{1}, \ldots, f_{k}$ locally cut out $X$ in $U$. We have $\Omega_{X}(U)=\Omega_{\mathbb{P}^{n}} \otimes \mathcal{O}_{X}(U) /\left(d f_{1}, \ldots, d f_{k}\right)$.

Firstly, let us verify (6). Pick an element $g \in I_{C / X}(U)$. Suppose we have

$$
d g=\sum_{j=1}^{n} \frac{\partial g}{\partial x_{j}} d x_{j}=0 \in \Omega_{X} \otimes \mathcal{O}_{C}(U) .
$$

There exist $a_{1}, \ldots, a_{k} \in \mathcal{O}_{C}(U)$ such that restricted to $C$,

$$
\frac{\partial g}{\partial x_{j}}=\sum_{i=1}^{k} a_{i} \frac{\partial f_{i}}{\partial x_{j}}, 1 \leq j \leq n .
$$

It follows that $d\left(g-\sum_{i=1}^{k} a_{i} f_{i}\right)=0$ on $C$. Since $C$ is smooth, the vanishing of $g-\sum_{i=1}^{k} a_{i} f_{i}$ and its differential on $C$ implies that $g-\sum_{i=1}^{k} a_{i} f_{i} \in I_{C}^{2}(U)$. Then we get $g=g-\sum_{i=1}^{k} a_{i} f_{i}=0$ as an element in $I_{C / X} / I_{C / X}^{2}(U)$.

Next, let us verify the exactness of (7). Take an element $h=\sum_{i=1}^{k} b_{i} f_{i} \in I_{X}(U)$. If $d h=0$ restricted to $C$, since $f_{1}, \ldots, f_{k}$ are vanishing on $C$, we have

$$
\sum_{i=1}^{k} b_{i} \frac{\partial f_{i}}{\partial x_{j}} d x_{j}=0,1 \leq j \leq n
$$

on $C$. Note that $X_{\text {sing }} \cap U$ consists of those points where the matrix

$$
\left(\frac{\partial f_{i}}{\partial x_{j}}\right)_{1 \leq i \leq k, 1 \leq j \leq n}
$$


drops rank. Since $C \cap X_{\text {sing }}$ consists of at most finitely many points, $b_{1}, \ldots, b_{k}$ must be vanishing at a non-empty open subset of $C \cap U$, which forces that they are vanishing completely on $C \cap U$. Hence, $h \otimes 1=\sum_{i=1}^{k} f_{i} \otimes b_{i}=0 \in\left(I_{X} / I_{X}^{2}\right) \otimes \mathcal{O}_{C}(U)$.

Now consider the deformation of $C$ on $X$. We have the following result.

Proposition 2.6. Keep the above assumption. If $C \cap X_{\text {sing }}$ is 0 -dimensional, the dimension of every component of $\mathrm{Hilb}^{d m+1-g}(X)$ at $[C]$ is at least

$$
\chi\left(N_{C / \mathbb{P} n}\right)-\chi\left(\left.N_{X / \mathbb{P}^{n}}\right|_{C}\right) .
$$

Moreover, suppose $X$ is a complete intersection cut out by hypersurfaces $F_{1}, \ldots, F_{k}$, $\operatorname{deg} F_{i}=d_{i}, i=1, \ldots, k$. The above lower bound can be written as

$$
\left(n+1-\sum_{i=1}^{k} d_{i}\right) d+(k-n+3)(g-1) .
$$

Proof. By the assumption, $C \subset X$ is generically unobstructed, so we can apply the result $[14$, Ch. $1,2.13,2.15]$. The local dimension of a component of $\operatorname{Hilb}^{d m+1-g}(X)$ at $[C]$ is at least

$$
\operatorname{dim} \operatorname{Hom}_{C}\left(I_{C / X} / I_{C / X}^{2}, \mathcal{O}_{C}\right)-\operatorname{dim} \operatorname{Ext}_{C}^{1}\left(I_{C / X} / I_{C / X}^{2}, \mathcal{O}_{C}\right)
$$

Note that if $X$ is smooth, the value of (8) is $\chi\left(N_{C / X}\right)$, which equals $\chi\left(N_{C / \mathbb{P}^{n}}\right)-$ $\chi\left(\left.N_{X / \mathbb{P}^{n}}\right|_{C}\right)$ due to the exact sequence

$$
\left.0 \rightarrow N_{C / X} \rightarrow N_{C / \mathbb{P}^{n}} \rightarrow N_{X / \mathbb{P}^{n}}\right|_{C} \rightarrow 0 .
$$

If $X$ is singular, apply the functor $\operatorname{Hom}\left(\cdot, \mathcal{O}_{C}\right)$ to $(6)$. Then we get a long exact sequence

$$
\begin{aligned}
0 & \rightarrow \operatorname{Hom}\left(\Omega_{C}, \mathcal{O}_{C}\right) \rightarrow \operatorname{Hom}\left(\Omega_{X} \otimes \mathcal{O}_{C}, \mathcal{O}_{C}\right) \rightarrow \operatorname{Hom}\left(I_{C / X} / I_{C / X}^{2}, \mathcal{O}_{C}\right) \\
& \hookrightarrow \operatorname{Ext}^{1}\left(\Omega_{C}, \mathcal{O}_{C}\right) \rightarrow \operatorname{Ext}^{1}\left(\Omega_{X} \otimes \mathcal{O}_{C}, \mathcal{O}_{C}\right) \rightarrow \operatorname{Ext}^{1}\left(I_{C / X} / I_{C / X}^{2}, \mathcal{O}_{C}\right) \\
\hookrightarrow &
\end{aligned}
$$

The last term is zero, because $\operatorname{Ext}^{2}\left(\Omega_{C}, \mathcal{O}_{C}\right)=H^{2}\left(T_{C}\right)=0$.

Moreover, apply the functor $\operatorname{Hom}\left(\cdot, \mathcal{O}_{C}\right)$ to $(7)$. We get another long exact sequence

$$
\begin{aligned}
& 0 \rightarrow \operatorname{Hom}\left(\Omega_{X} \otimes \mathcal{O}_{C}, \mathcal{O}_{C}\right) \rightarrow \operatorname{Hom}\left(\Omega_{\mathbb{P}} \otimes \mathcal{O}_{C}, \mathcal{O}_{C}\right) \rightarrow \operatorname{Hom}\left(\left(I_{X} / I_{X}^{2}\right) \otimes \mathcal{O}_{C}, \mathcal{O}_{C}\right) \\
& \hookrightarrow \operatorname{Ext}^{1}\left(\Omega_{X} \otimes \mathcal{O}_{C}, \mathcal{O}_{C}\right) \rightarrow \operatorname{Ext}^{1}\left(\Omega_{\mathbb{P} n} \otimes \mathcal{O}_{C}, \mathcal{O}_{C}\right) \rightarrow \operatorname{Ext}^{1}\left(\left(I_{X} / I_{X}^{2}\right) \otimes \mathcal{O}_{C}, \mathcal{O}_{C}\right) \\
& (10) \hookrightarrow \operatorname{Ext}^{2}\left(\Omega_{X} \otimes \mathcal{O}_{C}, \mathcal{O}_{C}\right) \rightarrow 0 .
\end{aligned}
$$

The last term is zero, because $\operatorname{Ext}^{2}\left(\Omega_{\mathbb{P}^{n}} \otimes \mathcal{O}_{C}, \mathcal{O}_{C}\right)=H^{2}\left(\left.T_{\mathbb{P}^{n}}\right|_{C}\right)=0$.

Note that $C$ is smooth, so $\operatorname{Ext}^{i}\left(\Omega_{C}, \mathcal{O}_{C}\right)=H^{i}\left(T_{C}\right)$ and $\operatorname{Ext}^{i}\left(\Omega_{\mathbb{P} n} \otimes \mathcal{O}_{C}, \mathcal{O}_{C}\right)=$ $H^{i}\left(\left.T_{\mathbb{P} n}\right|_{C}\right)$ for any $i$. From $(7)$, we know $\left(I_{X} / I_{X}^{2}\right) \otimes \mathcal{O}_{C}$ is locally free, so $\operatorname{Ext}^{i}\left(\left(I_{X} / I_{X}^{2}\right) \otimes \mathcal{O}_{C}, \mathcal{O}_{C}\right)=H^{i}\left(\left.N_{X / \mathbb{P}^{n}}\right|_{C}\right)$. Then by $(9)$ and (10), we have

$$
\begin{aligned}
& \operatorname{dim} \operatorname{Hom}\left(I_{C / X} / I_{C / X}^{2}, \mathcal{O}_{C}\right)-\operatorname{dim} \operatorname{Ext}^{1}\left(I_{C / X} / I_{C / X}^{2}, \mathcal{O}_{C}\right) \\
= & \chi\left(\left.T_{\mathbb{P}^{n}}\right|_{C}\right)-\chi\left(\left.N_{X / \mathbb{P}^{n}}\right|_{C}\right)-\chi\left(T_{C}\right)-\operatorname{dim} \operatorname{Ext}^{2}\left(\Omega_{X} \otimes \mathcal{O}_{C}, \mathcal{O}_{C}\right) \\
= & \chi\left(N_{C / \mathbb{P}^{n}}\right)-\chi\left(\left.N_{X / \mathbb{P}^{n}}\right|_{C}\right)-\operatorname{dim} \operatorname{Ext}^{2}\left(\Omega_{X} \otimes \mathcal{O}_{C}, \mathcal{O}_{C}\right) .
\end{aligned}
$$


For smooth curve $C, \chi\left(N_{C / \mathbb{P}^{n}}\right)$ equals $(n+1) d-(n-3)(g-1)$. If $X$ is a complete intersection cut out by $F_{1}, \ldots, F_{k}$, the normal sheaf $N_{X / \mathbb{P} n}$ splits into $\bigoplus_{i=1}^{k} \mathcal{O}_{X}\left(d_{i}\right)$. Therefore, in this case we can compute $\chi\left(\left.N_{X / \mathbb{P}^{n}}\right|_{C}\right)$ explicitly as

$$
\chi\left(\bigoplus_{i=1}^{k} \mathcal{O}_{C}\left(d_{i}\right)\right)=\sum_{i=1}^{k}\left(1-g+d d_{i}\right) .
$$

Now the proposition follows if we can show that $\operatorname{Ext}^{2}\left(\Omega_{X} \otimes \mathcal{O}_{C}, \mathcal{O}_{C}\right)=0$. In case $X$ is smooth, there is a short exact sequence

$$
0 \rightarrow T_{X} \otimes \mathcal{O}_{C} \rightarrow T_{\mathbb{P}^{n}} \otimes \mathcal{O}_{C} \rightarrow N_{X / \mathbb{P}^{n}} \otimes \mathcal{O}_{C} \rightarrow 0 .
$$

If $X$ is singular, the last map may not be surjective. Instead, we have

$$
0 \rightarrow T_{X} \otimes \mathcal{O}_{C} \rightarrow T_{\mathbb{P}^{n}} \otimes \mathcal{O}_{C} \rightarrow N_{X / \mathbb{P}^{n}} \otimes \mathcal{O}_{C} \rightarrow F \rightarrow 0,
$$

where $F$ is a sheaf supported at some points of $C \cap X_{\text {sing }}$. Split the above sequence into two short exact sequences

$$
\begin{gathered}
0 \rightarrow T_{X} \otimes \mathcal{O}_{C} \rightarrow T_{\mathbb{P}^{n}} \otimes \mathcal{O}_{C} \rightarrow E \rightarrow 0, \\
0 \rightarrow E \rightarrow N_{X / \mathbb{P}^{n}} \otimes \mathcal{O}_{C} \rightarrow F \rightarrow 0 .
\end{gathered}
$$

Since $H^{2}\left(T_{X} \otimes \mathcal{O}_{C}\right)=0$, then from (11), the map $H^{1}\left(T_{\mathbb{P}^{n}} \otimes \mathcal{O}_{C}\right) \rightarrow H^{1}(E)$ is surjective. Moreover, $F$ is only supported at finitely many points on $C$, so $H^{1}(F)=$ 0 . From (12), the map $H^{1}(E) \rightarrow H^{1}\left(N_{X / \mathbb{P} n} \otimes \mathcal{O}_{C}\right)$ is also surjective. Hence, we get a surjective map $H^{1}\left(T_{\mathbb{P}^{n}} \otimes O_{C}\right) \rightarrow H^{1}\left(N_{X / \mathbb{P}^{n}} \otimes \mathcal{O}_{C}\right)$, i.e. a surjective map $\operatorname{Ext}^{1}\left(\Omega_{\mathbb{P}^{n}} \otimes \mathcal{O}_{C}, \mathcal{O}_{C}\right) \rightarrow \operatorname{Ext}^{1}\left(\left(I_{X} / I_{X}^{2}\right) \otimes \mathcal{O}_{C}, \mathcal{O}_{C}\right)$ because $\Omega_{\mathbb{P}^{n}}$ and $I_{X} / I_{X}^{2}$ are both locally free. Then from (10), it follows that $\operatorname{Ext}^{2}\left(\Omega_{X} \otimes O_{C}, \mathcal{O}_{C}\right)=0$.

Now we can finish the proof of Theorem 1.3.

Proof of Theorem 1.3. For $g^{2} \geq d^{3}$, let us check that the smallest integer $s$ satisfying $s(s+1)<d$ and $g>\frac{d}{2}\left(s+\frac{d}{s+1}-3\right)$ is given by $s=\mu(d, g)$.

Rewrite the second inequality as

$$
d s^{2}-2(d+g) s+d^{2}-3 d-2 g<0 .
$$

Solve for $s$ and we get

$$
s>1+\frac{d^{2}-4 d-4 g}{g+\sqrt{g^{2}+4 d^{2}+4 d g-d^{3}}} .
$$

So the smallest integer $s$ satisfying this inequality is $\mu(d, g)$ by its definition.

In the range $d \sqrt{d} \leq g \leq \pi(d, 3)$, we have

$$
\mu(d, g)<2+\frac{d^{2}-4 g}{g} \leq \frac{d^{2}}{d \sqrt{d}}-2=\sqrt{d}-2 .
$$

Therefore, when $s=\mu(d, g)$, the inequality $s(s+1)<d$ also holds.

Apply Propositions 2.4 and 2.6 to the situation when $X=S$ is a surface of degree $\leq s$ in $\mathbb{P}^{3}$. The lower bound $4 d+g-1-s d$ obtained in (4) holds.

Now let us analyze this lower bound. Fix $d$ and view $\mu$ as a function of $g$ in the range $g^{2} \geq d^{3}$. In the expression of $\mu$, note that the numerator is decreasing and 
the denominator is increasing with $g$. So $\mu$ is decreasing when $g$ increases, hence the bound $4 d+g-1-\mu(d, g) d$ is increasing with $g$. Plugging in $g=d \sqrt{d}$, we get

$$
\mu \leq 2+\frac{d^{2}-4 d-4 d \sqrt{d}}{d \sqrt{d}} \leq \sqrt{d}-2,
$$

which implies that $g-1-\mu d>0$ when $g=d \sqrt{d}$. Therefore, for $g^{2} \geq d^{3}$ we always have $4 d+g-1-\mu(d, g) d>4 d$, i.e. $l_{d, g, 3}$ is strictly larger than the expected dimension $4 d$. Moreover, plugging in $g=\frac{d^{2}}{4}$ which is close to the Castelnuovo bound, we get $\mu \leq 2$. Correspondingly $l_{d, g, 3}>g+O(d)$ in this case, which has been already predicted by the Castelnuovo theory.

Below we will consider an example constructed in [3], which suggests that at least the order $d \sqrt{d}$ makes sense in Theorem 1.3.

Let $C$ be a space curve whose ideal sheaf has resolution as follows,

$$
0 \rightarrow O_{\mathbb{P}^{3}}^{\oplus s}(-s-1) \rightarrow O_{\mathbb{P}^{3}}^{\oplus(s+1)}(-s) \rightarrow I_{C} \rightarrow 0 .
$$

It is easy to derive the determinantal model for such a curve from this resolution. Pick an $s \times(s+1)$ matrix $A$ whose entries are general linear forms. Then the ideal sheaf of the curve defined by the determinants of all the $s \times s$ minors of $A$ has the above resolution. Tensor the exact sequence (13) with $\mathcal{O}_{\mathbb{P}^{3}}(k)$, and we get $h^{1}\left(I_{C}(k)\right)=0$ for any $k>0$. Hence, $C$ is projectively normal. We can also compute the Hilbert polynomial of $C$. When $k$ is large enough, we have

$$
\begin{aligned}
h^{0}\left(I_{C}(k)\right) & =(s+1) \cdot h^{0}\left(\mathcal{O}_{\mathbb{P}^{3}}(k-s)\right)-s \cdot h^{0}\left(\mathcal{O}_{\mathbb{P}^{3}}(k-s-1)\right) \\
& =\frac{1}{6}(k-s+2)(k-s+1)(k+2 s+3) .
\end{aligned}
$$

The Hilbert polynomial of $C$ equals

$$
h^{0}\left(O_{\mathbb{P}^{3}}(k)\right)-h^{0}\left(I_{C}(k)\right)=\frac{1}{2}\left(s^{2}+s\right) k-\frac{1}{6}\left(2 s^{3}-3 s^{2}-5 s\right) .
$$

So we obtain the degree and genus of $C$ :

$$
\begin{gathered}
d=\frac{1}{2} s(s+1), \\
g=1+\frac{1}{6}\left(2 s^{3}-3 s^{2}-5 s\right) .
\end{gathered}
$$

In [5, Thm. 1], it was proved that the locus of such curves $C$ is open inside the Hilbert scheme. Let $U$ be the corresponding Hilbert component that parameterizes such $C$. By [5, Thm. 2], $U$ has the expected dimension $4 d$. One can also count parameters to get the dimension of $U$ as

$$
4 s(s+1)-1-\operatorname{dim} \mathrm{PGL}_{s}-\operatorname{dim} \mathrm{PGL}_{s+1}=2 s^{2}+2 s=4 d .
$$

Note that as $s$ increases, $g^{2} \sim \frac{8}{9} d^{3}$.

The above construction does not cover all possible values of $d$ and $g$ in the range $g^{2}<d^{3}$. A more precise result was established in [15] as follows.

Theorem 2.7. For any $d, g$ such that $d \geq 20$ and $\frac{4}{3} d-4 \leq g \leq f(d)$ where $f(d)=$ $\frac{1}{6 \sqrt{2}} d \sqrt{d}+O(d)$, there exists a regular component of Hilb ${ }^{d m+1-g}\left(\mathbb{P}^{3}\right)$ with the expected dimension $4 d$. 
Note that asymptotically $g^{2} \sim \frac{1}{72} d^{3}$.

It would be interesting to figure out the remaining two questions.

Question 2.8. Does the expected dimension $4 d$ hold for some $g$ in the range $\frac{8}{9} d^{3}<$ $g^{2}<d^{3}$ ?

Question 2.9. What is the sharp leading coefficient of $f(d)$ such that a similar statement as in Theorem 2.7 still holds?

\section{The Hilbert scheme of curves in $\mathbb{P}^{4}$}

In this section we will prove Theorem 1.5. The idea of the proof is as follows. We will show that if $g$ is large enough, a degree $d$ genus $g$ smooth irreducible and nondegenerate curve $C$ in $\mathbb{P}^{4}$ must be contained in a surface $S$ such that $S$ is a complete intersection and $C$ is not contained in its singular locus $S_{\text {sing }}$. By estimating the dimension of the deformation of $C$ on $S$, we derive the desired result.

For the first step, let us recall some basic results from the Castelnuovo theory, cf. [9, Ch. 3].

Theorem 3.1. Let $C$ be a degree $d$ genus $g$ reduced irreducible and non-degenerate curve in $\mathbb{P}^{r}$. Then $g \leq \pi(d, r)=\frac{d^{2}}{2(r-1)}+O(d)$.

Now consider the case when $C$ is in $\mathbb{P}^{4}$. By the above theorem, it is easy to find a low degree threefold $F$ that contains $C$ if the genus $g$ is large enough.

Lemma 3.2. Let $k$ be a positive integer and $N=\left(\begin{array}{c}k+4 \\ 4\end{array}\right)-1$. If $g$ satisfies

$$
g>\pi(d k, N)
$$

then $C$ is contained in an irreducible threefold $F$ of degree $a \leq k$.

Proof. Embed $\mathbb{P}^{4}$ into $\mathbb{P}^{N}$ by the Veronese map of degree $k$. Then the image $C^{\prime}$ of $C$ is a curve of degree $d k$ and genus $g$. Since $g$ is larger than the Castelnuovo bound $\pi(d k, N), C^{\prime}$ must be contained in a hyperplane in $\mathbb{P}^{N}$. That is, $C$ is contained in a degree $k$ threefold in $\mathbb{P}^{4}$. Then we take an irreducible component $F$ of this threefold that contains $C$. Note that $F$ has degree $a \leq k$.

Fix $F$ and its degree $a$. Our next goal is to find another threefold that contains $C$ as well.

Lemma 3.3. Let $l \geq a$ be an integer. Let $M=\left(\begin{array}{c}l+4 \\ 4\end{array}\right)-\left(\begin{array}{c}l-a+4 \\ 4\end{array}\right)-1$. If $g$ satisfies

$$
g>\pi(d l, M)
$$

then there is another irreducible threefold $G$ of degree $b \leq l$ that contains $C$.

Proof. Embed $\mathbb{P}^{4}$ into $\mathbb{P}^{K}$ by the Veronese map of degree $l$, where $K=\left(\begin{array}{c}l+4 \\ 4\end{array}\right)$ 1. By a similar argument as before, we can show that $C$ is contained in at least $\left(\begin{array}{c}l-a+4 \\ 4\end{array}\right)+1$ independent degree $l$ threefolds in $\mathbb{P}^{4}$. Note that there are at most $\left(\begin{array}{c}l-a+4 \\ 4\end{array}\right)$ independent degree $l$ threefolds containing $F$ as a component, since $F$ is irreducible. Hence, we can find a degree $l$ threefold containing $C$ but not $F$. Take an irreducible component $G$ of this threefold that contains $C$. Then $G$ has degree $b \leq l$.

Let $S=F \cap G$ be the complete intersection surface that contains $C$. In order to apply deformation theory for $C \subset S$, we should avoid the situation $C \subset S_{\text {sing }}$. 
Lemma 3.4. Let $D$ be a reduced and connected curve of arithmetic genus $g$. If $D$ has $k$ irreducible components, then the number of singularities of $D$ is at most equal to $k+g-1$.

Proof. Let $\pi: \bar{D} \rightarrow D$ be the normalization of $D$. We have the short exact sequence

$$
0 \rightarrow \mathcal{O}_{D} \rightarrow \pi_{*} \mathcal{O}_{\bar{D}} \rightarrow \mathcal{F} \rightarrow 0
$$

where $\mathcal{F}$ is a sheaf supported on the singularities of $D$. The number of singularities of $D$ is bounded from above by $h^{0}(\mathcal{F})$. Moreover, from the long exact sequence of cohomology we have

$$
h^{0}(\mathcal{F}) \leq h^{0}\left(\pi_{*} \mathcal{O}_{\bar{D}}\right)+h^{1}\left(\mathcal{O}_{D}\right)-h^{0}\left(\mathcal{O}_{D}\right)=k+g-1 .
$$

The lemma follows right away.

Remark 3.5. Note that the above result is sharp. For instance, let $D$ be the union of $k$ general lines on a plane. The arithmetic genus of $D$ is $g=(k-1)(k-2) / 2$. The number of singularities of $D$ equals $\left(\begin{array}{l}k \\ 2\end{array}\right)$, which is exactly $k+g-1$.

Proposition 3.6. Keep the above assumption. If the degree $d$ of the curve $C$ satisfies

$$
d>\frac{1}{2} a b(a+b-2),
$$

then $C \cap S_{\text {sing }}$ is either empty or 0-dimensional. In particular, the dimension of the deformation of $C$ on $S$ is at least $5 d+g-1-(a+b) d$.

Proof. Take a general hyperplane section $X=H \cap S$ in $\mathbb{P}^{4}$. Note that $X$ is a curve of degree $a b$ and arithmetic genus $\frac{1}{2} a b(a+b-4)+1$ in $H \cong \mathbb{P}^{3}$. By Lemma 3.4, the total number of its singularities is at most $a b+\frac{1}{2} a b(a+b-4)+1-1=\frac{1}{2} a b(a+b-2)$. Since $H \cap S_{\text {sing }} \subset X_{\text {sing }}$, we have $\operatorname{deg} S_{\text {sing }} \leq \operatorname{deg} X_{\text {sing }} \leq \frac{1}{2} a b(a+b-2)$. Since the degree $d$ of $C$ is bigger than $\frac{1}{2} a b(a+b-2), C$ cannot be contained in $S_{\text {sing }}$.

Since $S$ is a complete intersection and $C \not \subset S_{\text {sing }}$, we can apply Proposition 2.6 to derive the desired estimate for the deformation of $C$ on $S$.

Now we can prove Theorem 1.5, which says that if $C$ is a degree $d$ genus $g$ smooth irreducible and non-degenerate curve in $\mathbb{P}^{4}$ such that $g>3 d \sqrt{d}+O(d)$, then $C$ is not rigid.

Proof of Theorem 1.5. In the range $g>3 d \sqrt{d}+O(d)$, we want to find integers $k, a, l, b$ successively in the above setting such that they satisfy the inequalities (14), (15) and (16). For simplicity, we only analyze leading terms, since the asymptotic orders are our main interests.

In Lemma 3.2, note that $N \sim \frac{k^{4}}{24}$. To satisfy the inequality (14), one can take $k \sim \frac{2 \sqrt{3} d}{\sqrt{g}}$. For a fixed number $a \leq k$, in Lemma 3.3 we have $M \sim \frac{l^{3} a}{6}$. To make the inequality (15) hold, we can choose $l \sim \frac{3 d^{2}}{a g}$. Now for a fixed number $b \leq l$, it suffices to verify the inequality (16). Note that $b$ can be at most equal to $l \sim \frac{3 d^{2}}{a g}$ and $g \geq \sim d \sqrt{d}$. These values maximize the right hand side of (16) as $\frac{1}{2} \sqrt{d}\left(a+\frac{\sqrt{d}}{a}\right)$, which is still less than $d$.

Therefore, by Lemmas 3.2, 3.3 and 3.4, we know that $C$ lies on a complete intersection surface $S$ of type $(a, b)$ and $C \not \subset S_{\text {sing }}$. Moreover, one can check that 
$(a+b) d<g$. Then by Proposition 3.6, the dimension of the deformation of $C$ on $S$ $\geq 5 d+g-1-(a+b) d \geq 5 d>24=\operatorname{dim} \operatorname{PGL}(5)$.

It is possible to enlarge the range $g>3 d \sqrt{d}+O(d)$ by refining the results in Lemmas 3.2, 3.3 and 3.4. However, it seems that only the leading coefficient could be improved rather than the exponent $d^{3 / 2}$. So when $g$ is slightly bigger than $d$, the situation remains mysterious to us. On the other hand, by the result of [2], Conjecture 1.6 in the introduction sounds highly possible and might be handled by an analogous argument. We state the conjecture again as the end of this section.

Conjecture 3.7. For $r \geq 5$, there always exists a constant $\lambda_{r}$ such that if $g \geq$ $\lambda_{r} d \sqrt{d}+O(d)$, a degree $d$ genus $g$ smooth irreducible and non-degenerate curve in $\mathbb{P}^{r}$ is not rigid.

\section{The Hilbert scheme of curves on a quadric threefold}

In this section we will prove Theorem 1.8. Recall that $Q$ is a smooth quadric threefold in $\mathbb{P}^{4}$. Let $U$ be an irreducible component of $\operatorname{Hilb}^{d m+1-g}(Q)$ whose general points parameterize smooth irreducible and non-degenerate curves in $Q$. The number $q_{d, g}$ is defined as the smallest dimension of all such components $U$. By the short exact sequence

$$
\left.0 \rightarrow N_{C / Q} \rightarrow N_{C / \mathbb{P}^{4}} \rightarrow N_{Q / \mathbb{P}^{4}}\right|_{C} \rightarrow 0,
$$

it follows that $\chi\left(N_{C / Q}\right)=3 d$. Hence, $3 d$ is a lower bound for $q_{d, g}$. Theorem 1.8 provides a further analysis for the sharpness of this bound. It consists of two parts.

Firstly, if $g$ is large enough, $C$ must lie on a threefold $F$ of low degree, which does not contain $Q$ as a component. By considering the deformation of $C$ on the surface $X=Q \cap F$, we derive the first part of Theorem 1.8. For the second part, we use a similar method as in [15]. A component whose general elements parameterize a curve as the intersection of $Q$ and a determinantal surface has dimension $3 d$. Then we apply the smoothing technique in [16] to enlarge the range of the pair $(d, g)$ to cover the case when $g<\frac{2}{15 \sqrt{5}} d \sqrt{d}+O(d)$.

Proof of Theorem 1.8 (1). Let $k$ be the largest integer satisfying $d>2 k(k-1)$. Since $k \sim \sqrt{\frac{d}{2}}$, the inequality $g>\frac{d^{2}}{4 k}+\frac{1}{2} k d$ holds in the range $g>\frac{1}{\sqrt{2}} d \sqrt{d}+O(d)$. By $[1$, Thm. 1.4], there exists an integral surface $X \in\left|O_{Q}(a)\right|$ containing $C$, where $a \leq k$. Since $d>2 k(k-1)$ and $X$ is of degree $2 a$, by Proposition 3.6 we have $C \not \subset X_{\text {sing }}$. Then by Proposition 2.6, $\chi\left(N_{C / X}\right)=3 d+g-a d-1$ provides a lower bound for the dimension of the deformation of $C$ on $X$. Note that $3 d+g-a d-1 \geq 3 d+g-k d-1>3 d$ since $k \sim \sqrt{\frac{d}{2}}$ and $g>\frac{1}{\sqrt{2}} d \sqrt{d}+O(d)$.

The second part is more complicated. We want to construct a component of the Hilbert scheme that parameterizes certain determinantal curves. But those curves have to be contained in the quadric threefold $Q$. A natural idea is to take the intersection of a determinantal surface with $Q$.

Let $\left(H_{i j}\right)$ be a $t \times(t+1)$ general matrix, whose entry $H_{i j}$ is a general linear form in $\mathbb{P}^{4}$. Its $t \times t$ minors define a determinantal surface $S$. The ideal sheaf of $S$ has the following resolution

$$
0 \rightarrow \mathcal{O}_{\mathbb{P}^{4}}^{\oplus t}(-t-1) \rightarrow \mathcal{O}_{\mathbb{P}^{4}}^{\oplus(t+1)}(-t) \rightarrow I_{S} \rightarrow 0 .
$$


By Bertini, if we take a general quadric threefold $Q$, then $C=Q \cap S$ is smooth. From the above exact sequence, it is not hard to obtain the degree and genus of $C$ :

$$
\begin{gathered}
d=t(t+1), \\
g=\frac{2}{3} t^{3}-\frac{1}{2} t^{2}-\frac{7}{6} t+1 .
\end{gathered}
$$

Note that asymptotically $g \sim \frac{2}{3} d \sqrt{d}$.

Moreover, for general $S$ and $Q$, the ideal sheaf $I_{C / Q}$ has the resolution

$$
0 \rightarrow \mathcal{O}_{Q}^{\oplus t}(-t-1) \rightarrow \mathcal{O}_{Q}^{\oplus(t+1)}(-t) \rightarrow I_{C / Q} \rightarrow 0 .
$$

By $\left[13\right.$, Rmk. 2.2.6], we know that $H^{1}\left(N_{C / Q}\right)=\operatorname{Ext}_{Q}^{2}\left(I_{C / Q}, I_{C / Q}\right)$. Apply the functor $\operatorname{Hom}_{Q}\left(-, I_{C / Q}\right)$ to the above exact sequence. One can derive that $H^{1}\left(N_{C / Q}\right)=0$, which implies that there is no obstruction for the deformation of $C$ on $Q$. Hence, $h^{0}\left(N_{C / Q}\right)=\chi\left(N_{C / Q}\right)=3 d$ is the exact dimension for the component of Hilb $^{d m+1-g}(Q)$ that contains $C$.

The above construction is nice. But it has strong restriction on the values of $d$ and $g$. We would like to extend the result to more general values of $d$ and $g$. Here we will follow the methods in [15] and [16]. The idea works as follows. Take a smooth determinantal curve $\Gamma$ constructed as above and a smooth rational curve $\gamma$ on $Q$ such that they meet transversely. Further assume that $H^{1}\left(N_{\Gamma / Q}\right)=H^{1}\left(N_{\gamma / Q}\right)=0$. Then the nodal curve $\Gamma \cup \gamma$ can be smoothed out on $Q$. Moreover, the vanishing property of $H^{1}(N)$ is locally preserved under this smoothing process. Then after smoothing the nodal curve, we can obtain the degree and genus in a more general range.

Firstly, let us adapt to our situation a smoothing technique from [16].

Lemma 4.1. Let $\Gamma^{\prime}=\Gamma \cup \gamma$ be a nodal union of two smooth and irreducible curves on the quadric threefold $Q$. Assume that $\Gamma \cap \gamma=P_{1}, \ldots, P_{\delta}$. If $H^{1}\left(N_{\Gamma / Q}\right)=H^{1}\left(N_{\gamma / Q}\right)=$ $H^{1}\left(N_{\gamma / Q}\left(-P_{1}-\ldots-P_{\delta}\right)\right)=0$, then $H^{1}\left(N_{\Gamma^{\prime} / Q}\right)=0$ and $\Gamma^{\prime}$ is smoothable on $Q$.

Proof. Let us first set up some notation. For a connected reduced curve $C$ on $Q$, denote $N_{C / Q}^{\prime}$ as the cokernel of the map $T_{C} \rightarrow T_{Q \mid C}$ and let $T_{C / Q}^{1}$ be the cotangent sheaf of $C$ on $Q$. Note that $T_{C / Q}^{1}$ can be defined as the cokernel of the map $N_{C / Q}^{\prime} \rightarrow$ $N_{C / Q}$. Suppose the singularities of $C$ are only nodes. Then $T_{C / Q}^{1}$ is a torsion sheaf supported on each node of $C$. Furthermore, if $H^{1}\left(N_{C / Q}^{\prime}\right)=0$, by the argument of [16, Prop. 1.6], $C$ is smoothable on $Q$.

Now in our case, the ideal sheaves $I_{\Gamma / \Gamma^{\prime}} \cong \mathcal{O}_{\gamma}\left(-P_{1}-\ldots-P_{\delta}\right)$ and $I_{\gamma / \Gamma^{\prime}} \cong \mathcal{O}_{\Gamma}\left(-P_{1}-\right.$ $\left.\ldots-P_{\delta}\right)$. As in $\left[16\right.$, Lem. 5.1], we can establish two exact sequences of sheaves on $\Gamma^{\prime}$,

$$
\begin{aligned}
0 \rightarrow I_{\Gamma / \Gamma^{\prime}} \otimes N_{\Gamma^{\prime} / Q} & \rightarrow N_{\Gamma^{\prime} / Q}^{\prime} \rightarrow N_{\Gamma / Q} \rightarrow 0, \\
0 \rightarrow N_{\gamma / Q}\left(-P_{1}-\ldots-P_{\delta}\right) & \rightarrow I_{\Gamma / \Gamma^{\prime}} \otimes N_{\Gamma^{\prime} / Q} \rightarrow T_{\Gamma^{\prime} / Q}^{1} \rightarrow 0 .
\end{aligned}
$$

By the assumption and the fact that $H^{1}\left(T_{\Gamma^{\prime} / Q}^{1}\right)=0$, we get $H^{1}\left(N_{\Gamma^{\prime} / Q}^{\prime}\right)=0$ from the above long exact sequences of cohomology. Hence, $\Gamma^{\prime}$ is smoothable on $Q$. Moreover, the map $N_{\Gamma^{\prime} / Q}^{\prime} \rightarrow N_{\Gamma^{\prime} / Q}$ is injective and its cokernel $T_{\Gamma^{\prime} / Q}^{1}$ is supported at the nodes. So $H^{1}\left(N_{\Gamma^{\prime} / Q}^{\prime}\right)=0$ implies that $H^{1}\left(N_{\Gamma^{\prime} / Q}\right)=0$.

We still need another source curve $\gamma$. Here we will consider rational curves that lie on the quadric $Q$. 
Lemma 4.2. Let $R=Q \cap H$ be a general hyperplane section of $Q$. Let $P_{1}, \ldots, P_{m} \in R$ denote $m \geq 6$ points in general position. For any integer $\delta \leq 4$, there exists a degree three rational curve $\gamma \subset R$ such that $\gamma$ passes through exactly $\delta$ points of $P_{1}, \ldots, P_{m}$. Furthermore, suppose those points on $\gamma$ are $P_{1}, \ldots, P_{\delta}$. Then we have $H^{1}\left(N_{\gamma / Q}\left(-P_{1}-\ldots-P_{\delta}\right)\right)=0$.

Proof. $R$ is a smooth quadric surface in $H \cong \mathbb{P}^{3}$. It is easy to find a degree 3 smooth rational curve $\gamma$ on $R$ that passes through $\delta$ general points, say, $P_{1}, \ldots, P_{\delta}$. There is an exact sequence

$$
0 \rightarrow N_{\gamma / R} \rightarrow N_{\gamma / Q} \rightarrow N_{R / Q} \otimes \mathcal{O}_{\gamma} \rightarrow 0
$$

Moreover, we know that $N_{R / Q} \otimes \mathcal{O}_{\gamma}$ and $N_{\gamma / R}$ have degree 3 and 4 on $\gamma \cong \mathbb{P}^{1}$, respectively. Tensor the above exact sequence with $\mathcal{O}_{\gamma}\left(-P_{1}-\ldots-P_{\delta}\right)$ and we get

$$
0 \rightarrow \mathcal{O}_{\mathbb{P}^{1}}(4-\delta) \rightarrow N_{\gamma / Q}\left(-P_{1}-\ldots-P_{\delta}\right) \rightarrow \mathcal{O}_{\mathbb{P}^{1}}(3-\delta) \rightarrow 0 .
$$

Since $\delta \leq 4$, it follows that $H^{1}\left(N_{\gamma / Q}\left(-P_{1}-\ldots-P_{\delta}\right)\right)=0$.

Now we have all the ingredients to prove the second part of Theorem 1.8.

Proof of Theorem 1.8 (2). Take a determinantal curve $\Gamma \subset Q$ of degree $d_{\Gamma}=t(t+1)$ and genus $g_{\Gamma}=\frac{2}{3} t^{3}-\frac{1}{2} t^{2}-\frac{7}{6} t+1$. Consider a general hyperplane section of $\Gamma$. We get $d_{\Gamma}$ points in general position. By Lemma 4.1 and 4.2 , we can take a suitable degree 3 rational curve $\gamma$, such that $\Gamma^{\prime}=\Gamma \cap \gamma$ consists of $\delta$ reduced points for any $\delta \leq 4$. In particular, $\Gamma^{\prime}$ is smoothable and $H^{1}\left(N_{\Gamma^{\prime} / Q}\right)=0$. Then there is a Hilbert component containing $\Gamma^{\prime}$ and certain smooth curves as its general points arising from the smoothing of $\Gamma^{\prime}$. This component has dimension $3 d$, since its tangent space at $\left[\Gamma^{\prime}\right]$ has dimension equal to $h^{0}\left(N_{\Gamma^{\prime} / Q}\right)=\chi\left(N_{\Gamma^{\prime} / Q}\right)=3 d$. Hence, starting from the pair $\left(d_{\Gamma}, g_{\Gamma}\right)$, we can get a new pair $\left(d^{\prime}=d_{\Gamma}+3, g^{\prime}=g_{\Gamma}+\delta-1\right)$ where the Hilbert scheme Hilb $^{d^{\prime} m+1-g^{\prime}}(Q)$ has a component of expected dimension $3 d^{\prime}$. Do the same step again and eventually it will cover every pair $(d, g)$ of type $\left(d_{\Gamma}+3 k, g_{\Gamma}+h\right), 0 \leq h \leq 3 k$.

Now we fix $d$. Note that $d_{\Gamma}=t(t+1) \equiv 0$ or $2(\bmod 3)$. So if $d \equiv 0(\bmod 3)$, by the above construction, the range of $g$ for which Hilb ${ }^{d m+1-g}(Q)$ has a component of dimension $3 d$ contains the following,

$$
\frac{1}{6}\left(4 t^{3}-3 t^{2}-7 t+6\right) \leq g \leq \frac{1}{6}\left(4 t^{3}-3 t^{2}-7 t+6\right)+3 \cdot \frac{d-t(t+1)}{3},
$$

for any $t(t+1) \leq d$ and $t \equiv 0$ or $2(\bmod 3)$. In order to cover the case $d \equiv 1$ or 2 $(\bmod 3)$, we can use a suitable line $l$ on $Q$ instead of the rational curve $\gamma$ in Lemma 4.2 such that $l$ intersects the source curve only at one point $P$. One can easily check that $H^{1}\left(N_{l / Q}\right)=H^{1}\left(N_{l / Q}(-P)\right)=0$ holds. Then after smoothing the nodal union of $l$ and the source curve, this construction provides $(d-1, g) \rightarrow(d, g)$. So if $d \not \equiv 0$ ( $\bmod 3)$, we can always consider $d-1$ or $d-2$ instead. In sum, the desired range of genus includes

$$
L(t)=\frac{1}{6}\left(4 t^{3}-3 t^{2}-7 t+6\right) \leq g \leq \frac{1}{6}\left(4 t^{3}-3 t^{2}-7 t+6\right)+d-t(t+1)-2=R(t),
$$

where $t(t+1) \equiv 0(\bmod 3)$. Since $t \equiv 0$ or $2(\bmod 3)$, each time $t$ increases by 1 or 2 . In order to avoid that the value of $g$ jumps for a fixed $d$, we have to require that $L(t+2) \leq R(t)$. Solving this inequality and plugging the upper bound of $t$ into $R(t)$, we get the desired range of $g$ up to $\frac{2}{15 \sqrt{5}} d \sqrt{d}+O(d)$. 
Remark 4.3. We can obtain a similar result for the Hilbert scheme of curves on a general cubic threefold $Y$. One can check that the curve $C$ cut out by a determinantal surface with $Y$ satisfies $H^{1}\left(N_{C / Y}\right)=0$. However, when we resume the process to quartic threefolds, the determinantal model does not work any more. Another long standing problem is about quintic threefolds, since the expected dimension of the Hilbert scheme is zero in that case. Even for rational curves, the famous Clemens' conjecture has been only solved when the degree of the curve is small. If we consider threefolds of higher degree, things become further unclear. To the author's best knowledge, we even do not know if a general threefold of degree $k>5$ in $\mathbb{P}^{4}$ contains an irreducible curve whose degree is not divisible by $k$. In sum, the Hilbert scheme of curves on a threefold of higher degree remains mysterious to us.

\section{Acknowledgements}

The author is grateful to Joe Harris, who first told him about this question and made numerous suggestions during the preparation of this work. The author would also like to thank the referee for many excellent comments on the details and structure of this paper.

\section{Bibliography}

[1] M. A. A. De Cataldo, The genus of curves on the three-dimensional quadric, Nagoya Math. J. 147 (1997), 193-211.

[2] L. Chiantini, C. Ciliberto and V. Di Gennaro, The genus of projective curves, Duke Math. J. 70 (1993), no. 2, 229-245.

[3] P. Ellia, Exemple de courdes de $\mathbb{P}^{3}$ à fibré normal semi-stable, stable, Math. Ann. 264 (1983), 389-396.

[4] P. Ellia, A. Hirschowitz and E. Mezzetti, On the number of irreducible components of the Hilbert scheme of smooth space curves, Internat. J. Math. 3 (1992), no. 6, 799-807.

[5] G. Ellingsrud, Sur le Schéma de Hilbert des variétés de codimension 2 dans $\mathbb{P}^{e}$ à cône de Cohen-Macaulay, Ann. Sci. école Norm. Sup. (4) 8 (1975), no. 4, 423-431.

[6] G. Fløystad, Construction of space curves with good properties, Math. Ann. 289 (1991), 33-54.

[7] A. Grothendieck, Techniques de construction et théorèmes dexistence en géometrie algébrique, IV: Les schémas de Hilbert. In Seminaire Bourbaki, 1960-61, Exposés 205-222, Exposé 221, 221.1-221.28 (New York: Benjamin), 1966.

[8] L. Gruson, C. Peskine, Genre des courbes de l'espace projectif, Algebraic geometry (Proc. Sympos., Univ. Troms $\varnothing$, Troms $\varnothing, 1977), 31-59$, Lecture Notes in Math. 687, Springer, Berlin, 1978.

[9] J. Harris, Curves in projective spaces, Montréal, Les Presses de L’Université de Montreal, 1982.

[10] J. Harris and I. Morrison, Moduli of curves, Springer, 1998.

[11] R. Hartshorne, Connectedness of the Hilbert scheme, Publ. Math. I.H.E.S., 29 (1966), 5-48.

[12] R. Hartshorne, Algebraic geometry, Spring-Verlag, 1977.

[13] J. O. Kleppe, The Hilbert-flag scheme, its properties and its connection with the Hilbert scheme, Ph.D. Thesis, University of Oslo, 1981.

[14] J. Kollár, Rational curves on algebraic varieties, Springer-Verlag, 1996.

[15] G. Pareschi, Components of the Hilbert scheme of smooth space curves with the expected number of moduli, Manuscripta Math. 63 (1989), 1-16.

[16] E. Sernesi, On the existence of certain families of curves, Invent. Math. 75 (1984), 25-57.

University of Illinois at Chicago, Department of Mathematics, Statistics and Computer Science, Chicago, IL 60607

E-mail address: dwchen@math.uic.edu 